\title{
Clinical Application of Soft Ureter Mirror
}

\author{
Fan-Ping MENG ${ }^{1, a}$, Yong-liang CUI ${ }^{1}$, Jun $\mathrm{WEI}^{1}$ and Ji GAO ${ }^{1, \mathrm{~b},{ }^{*}}$ \\ ${ }^{1}$ Department of Urology, China-Japan Union Hospital, Jilin University, Changchun, \\ Jilin, China \\ a947410581@qq.com, b1217760@qq.com \\ ${ }^{*}$ Corresponding author
}

Keywords: Soft ureter mirror, Kidney stones, Lithotripsy.

\begin{abstract}
Objective:To summarize the clinical application of ureter soft mirror. Methods:The treatment of urinary calculi includes ESWL, percutaneous nephrolithotomy, ureter soft mirror and so on , The treatment of urinary calculi with ureter soft mirror is becoming more and more widespread, and the indications are constantly relaxed. The stone size is no longer limited to less than $2 \mathrm{~cm}$ of kidney stones. In addition to urinary calculi, ureter soft mirror in the Department of Urology in other areas of its application value, including UPJ stenosis of internal incision, the removal of endogenous renal cyst, superficial tumor laser resection, Diagnosis of hematuria, etc.. Conclusion: Ureteral urology clinical application of soft lens technology in our country is still in its initial stage of groping requires more independent innovation and standardize the clinical and scientific research of technical equipment, promote ureteral soft lens surgery of conduct, to further improve the level of urology minimally invasive treatment in China.
\end{abstract}

\section{Introduction}

Soft ureter mirror has the characteristics of minimally invasive, safe and effective, is being more and more domestic urological surgeons used and valued ${ }^{[1]}$.Developed countries in Europe and the us soft ureter mirror technology is an important part of urology minimally invasive treatment technology, is widely of conducted clinical application;In recent years, the soft ureter mirror in our country is becoming more and more widely.

\section{Methods}

The main means of minimally invasive treatment including kidney stones extracorporeal shock wave lithotripsy (ESWL), soft ureter mirror, percutaneous nephroscope (PCNL), and other ways ${ }^{[2]}$.Although the majority of kidney and ureter calculus by ESWL treatment, but the size of the localization, the stones and inflammatory reaction of the chemical composition, stop positions, polyps, and stone obstruction, and patients with obese due to the influence of such factors as rock failure. PCNL therapy on kidney and ureter Duan Jieshi curative effect, but the operation channel through the renal parenchyma, complex operation, traumatic to the kidney is larger, intraoperative bleeding, postoperative complications such as sepsis, hemopneumothorax and surrounding tissue injury, serious and even death in patients with renal resection.Michel reported PCNL various complication rates as high as $83 \%$.Soft and ureter mirror at the same time are effective, minimally invasive and less 
complications, to merge with ureteral stricture, renal pelvis ureter connection (UPJ) stricture or combination of polyps can be cut and removed.

Stone size and position are the main factors influencing the treatment choice $^{[3]}$.Kidney stones for less than or equal to $20 \mathrm{~mm}$, the domestic and foreign scholars reported soft lens with PCNL ureter calculi clearance rate basically the same, and the operation risks of soft lens more advantage; The controversial for more than 20 $\mathrm{mm}$ of kidney stones, stones more than $20 \mathrm{~mm}$ in diameter operation time may be longer, increasing the incidence of kidney damage and infection, at the same time will increase the wastage of the soft lens. Hussain, soft ureter mirror treatment of kidney stone diameter less than or equal to $20 \mathrm{~mm}$ single gravel success rate of $96.5 \%$, and a diameter greater than $20 \mathrm{~m}$ group single gravel success rate is only $58.3 \%$. Takazawa applications such as soft ureter mirror treatment of 20 patients with kidney stones in 2 $5 \mathrm{~cm}$ in diameter, single stone clearance rate is only $65 \%$.In addition some scholars believe that although an operation calculi clearance rate of PCNL currently has the edge, but soft ureter mirror has small trauma, the advantages of good security and can repeat treatment, $20 \mathrm{~mm}$ in diameter kidney ureteral calculi antler shape stones, even staging many times soft ureter mirror gravel also can obtain good curative effect. Such as Hyams report stage surgical treatment of 120 cases of ureteral soft mirror size is $2 \sim 3 \mathrm{~cm}$ kidney stones, II rubble after the operation, the success rate of $95.7 \%$. xiaofeng gao, such as review of treatment of 99 cases of ureteral soft mirror $2 \sim 4 \mathrm{~cm}$, kidney stones after II period operation has achieved $94.9 \%$ stone clearance, think in installment soft ureter mirror surgical treatment for $2 \sim 4 \mathrm{~cm}$ safe, kidney stones and effective, can be selectively as PCNL alternatives. The position of the stones in the kidney determines the success rate of the soft lens of gravel clearance significantly below the upper calyx calculi under light, the study found that soft ureter mirror treatment success rate of renal calyx calculi under main and renal pelvis below calyx Angle, light funnel the anatomical factors such as length and width. Geavlete etc. The study found that renal pelvis and ureter under light Angle is greater than $90^{\circ}$, soft ureter mirror lithotripsy success rate was $87.5 \%, 30^{\circ} \sim 90^{\circ}$ was $74.3 \%,<30^{\circ}$ is $0 \%$; Renal pelvis and ureter under light Angle of $30^{\circ} \sim 90^{\circ}$, the minister of subgroup analysis found that the calyx funnel degree is less than $3 \mathrm{~cm}$ group contrast is greater than $3 \mathrm{~cm}$, gravel success rate are $88.2 \%$ and $88.2 \%$ respectively, the difference was statistically significant.

It is the first choice for treatment of ureteral calculi ESWL, ureteroscopy especially soft lens but also high security.Hard for lower ureter Duan Jieshi, ureter mirror is also a main treatment method, soft ureter mirror for can effectively handle gravel up or return the kidney stones, in the process of Duan Jieshi has more advantages in the ureter.33 Kijvikai system analysis such as the ESWL with ureteroscopy on the treatment of ureteral Duan Jieshi literature, less than $10 \mathrm{~mm}$ group calculi clearance rate were $86 \%$, $90 \%$, more than $10 \mathrm{~mm}$ group calculi clearance rate were $67 \%, 73 \%$, think less than 10 $\mathrm{mm}$ on ureteral Duan Jieshi ESWL is a reasonable choice, choose ureteral greater than $10 \mathrm{~mm}$ lens can get the best stone clearance rate. XIAN LI, such as new use soft lens processing segment ureteral calculi achieved $91.7 \%$ success rate of gravel.In addition, soft ureter mirror also suitable for ESWL, PCNL and even failure of the open surgery in the treatment of urinary stones;Soft Cocuzza applications such as ureter mirror treatment after failed ESWL ureteral Duan Jieshi achieve good curative effect.Poor curative effect for ESWL is expected or invalid, ureter Duan Jieshi on more than $10 \mathrm{~mm}$ in diameter and diameter less than or equal to $20 \mathrm{~mm}$ of kidney stones, soft lens can be used as the preferred treatment, renal calyx calculi under should be considered under the renal pelvis and ureter anatomical factors such as light Angle.PCNL is a standard way 
of treatment is greater than $2 \mathrm{~cm}$ kidney stones, but good repeatability of ureteral soft lens can be used as more than $20 \mathrm{~mm}$ the alternatives of kidney stones, can also be used to deal with the postoperative residual stone of PCNL.

Soft ureter mirror treatment of urinary calculi in widening the scope of indication as for special groups such as merger hemorrhagic physique, obese, pregnant women, infants and young children stones and some special kidney stones such as calyces diverticulum stones, polycystic kidney, kidney, repeat renal, pelvic ectopic kidney, horseshoe kidney, medullary sponge kidney position deformity or a renal anatomy of kidney stones, ESWL, PCNL usually poor efficacy or unable to apply, the soft ureter mirror showed obvious superiority.

Turna reports such as kidney stones patients if need of anticoagulant drugs but can't scratch, not completely normal coagulation function, for soft ureter mirror qin laser lithotripsy is safe, does not increase bleeding complications ${ }^{[4]}$. Natalin using soft ureter mirror lithotrity treatment such as normal quality, super quality and obesity on the three groups of patients with urinary stones, gravel success rate has no obvious difference between them, that soft ureter mirror lithotripsy is not affected by the degree of obesity.Soft ureter mirror in urinary stones on the treatment of children with high efficiency, and less complications; Kim, etc and Nerli with soft lens on treating children urinary stones has achieved success rate of more than $97 \%$ of the rubble, and no obvious complications. ChuYingHao adopts soft lens treatment of 9 cases of infant melamine stone also achieve good curative effect.On the part of the pregnancy symptoms after conservative symptomatic treatment patients with urinary stones can't ease still need surgical intervention. Ishii, such as system reviewed nearly 23 years ureteroscopy treatment during pregnancy urinary stones on the related literature, the results showed that urinary calculi ureteroscopy treatment of the pregnant woman is safe, effective;Jiang Xian states such as using soft ureter mirror treatment of 9 cases of ureteral calculi during pregnancy success entirely, and no complications occurred. CHUN YANG klosterman and using ureteral soft lens 23 cases with clinical symptoms of calyces diverticulum calculi with $87 \%$ success rate of gravel, no complications, soft ureter mirror can be used as the first selection of calyces diverticulum stone processing. Weizer application soft ureter mirror processing such as pelvic ectopic kidney, horseshoe kidney stones, stone clearance rate was $87.5 \%$, and no obvious complications occurred, soft ureter mirror can be used as such anatomical, location deformity in the treatment of choice for kidney stones. Autosomal dominant polycystic kidney with patients with kidney stones, because multiple cyst compression kidney calyces space is narrow, PCNL processing difficulties, such as Liu reported in 13 cases of urinary stones on patients with polycystic kidney ureteral soft lens lithotripsy treatment, gravel success rate was $92.3 \%$, and the curative effect is distinct, no severe complications.

In soft ureter mirror complications, postoperative infection is in the most serious is the most common situation.Infection with the length of time of surgery, intraoperative perfusion wash the renal pelvis and pressure and so on.If existing preoperative urinary tract infections, need to control infection after undergoing elective surgery;For large calculi surgery by stages shorten the operation time also can reduce the incidence of infection;Rubble when maintaining low pressure is also the key to prevent infection in renal pelvis, and intraoperative placed outside the ureter sheath, can help keep intraoperative drainage unobstructed, reduce pressure in the renal pelvis.Preoperative 1 $\sim 2$ weeks D-J tube placement can improve the success rate of ureter sheath placement.Intraoperative control flushing pressure also can reduce the incidence of 
postoperative infection.Chinese scholars reported purely retrograde soft in the treatment of urinary calculi surgery is $1.79 \%$ (104/5822) overall incidence of serious complications, including postoperative infection with high fever 22 cases, including 9 cases of septic shock and deterioration of renal function, 4 cases of ureteral avulsion/perforation ( 3 cases), ureteral stone street 38 cases, severe bleeding or 25 cases of blood in the urine, a large amount of urinary extravasation in 1 case, renal subcapsular hematoma in 3, edema, 8 cases of renal week.

At present the use of expensive and the maintenance cost is soft ureter mirror technology in the domestic application of the biggest obstacles.Germany in recent years, platinum set company production of the new combination of detachable soft ureter mirror used in clinical, due to its main core parts removable, can partly solve soft one-piece ureter mirror damaged problem of main parts, to a certain extent, reduce the use of soft ureter mirror and the maintenance cost.

\section{Conclusions}

In general, the introduction of more than 20 years to soft ureter mirror has relative popularity at home, but a serious shortage of clinical conduct since.Applied to the upper tract tumor, especially in the early diagnosis and treatment of renal pelvis carcinoma stone less illness.Ureteral urology clinical application of soft lens technology in our country is still in its initial stage of groping requires more independent innovation and standardize the clinical and scientific research of technical equipment, promote ureteral soft lens surgery of conduct, to further improve the level of urology minimally invasive treatment in China.

\section{References}

[1] The safety of ureteroscopy during pregnancy:a systematic review and meta-analysis. SEMINS MJ, TROCK BJ, MATLAGA BR. Journal d Urologie, 2009.

[2] Flexible ureterorenoscopy: prospective analysis of the Guy's experience. Dasgupta P, Cynk MS, Bultitude MF, et al. Annals of the Royal College of Surgeons of England, 2004.

[3] Ureteroscopic management of renal calculi in anomalous kidneys[J] . Alon Z. Weizer,W. Patrick Springhart,Wesley O. Ekeruo,Brian R. Matlaga, Yeh H. Tan, Dean G. Assimos, Glenn M. Preminger. Urology. 2005 (2)

[4] Efficacy of retrograde ureteropyeloscopic holmium laser lithotripsy for intrarenal calculi $>2 \mathrm{~cm}[\mathrm{~J}]$. M. J. Bader,C. Gratzke, S. Walther, P. Weidlich, M. Staehler, M. Seitz, R. Sroka ,O. Reich, C. G. Stief, B. Schlenker. Urological Research. 2010 (5) 